\title{
INTEGRACIÓN METODOLÓGICA Método de análisis de la imagen de las organizaciones deportivas en el deporte escolar en la comarca Ribera Baja del Ebro
}

\section{Autores}

Dr. Alberto Nuviala Nuviala

Facultad Ciencias de la Actividad Física y el Deporte Facultad Ciencias de la Actividad Fisica y el Deporte Universidad de Pablo de Olavides de Sevilla
Dr. Francisco Ruiz Juan

Facultad de Humanidades y Ciencias de la Educación

Universidad de Almería

\section{Resumen}

El marco jurídico otorga las competencias a las diferentes organizaciones para desempeñar la prestación de un servicio. Conocerlo ayuda a determinar la responsabilidad de cada una, simultáneamente permite coordinar sus acciones. Los usuarios del servicio valoran esta prestación al tiempo que se hacen una imagen de la organización. El objeto de este trabajo es conocer la imagen que niños, padres y técnicos deportivos tiene de las organizaciones con competencias en el deporte escolar. Para ello hemos utilizado la integración metodológica materializada a través del cuestionario y el grupo de discusión. Los resultados demuestran la escasa valoración, por parte de los padres, de las organizaciones, mientras que los técnicos aprueban con mayor calificación a las organizaciones públicas más próximas a los usuarios.

Palabras clave: Competencias, organizaciones, imagen, deporte escolar.

\section{Competencias de las} orgorganizaciones aragonesas en el deporte escolar

La imagen de una organización no sólo se construye a través de la comunicación externa, sino que a ella contribuye, y en gran medida, la imagen corporativa. Éste es el modo en que el público, los usuarios del servicio, perciben la organización a través de su forma de actuación y a través de los recursos, humanos y materiales que utiliza para el desempeño del servicio. La imagen es la valoración del usuario, ciudadano o cliente de la organización, en la que intervienen factores muy diversos: filosofía de la organización, historia, cultura, estilo de gestión, conducta de empleados..

Para incidir en la imagen de las organizaciones con competencias en la prestación del servicio deporte escolar debe actuarse sobre el total de la organización, debiendo asociarse la prestación del servicio a una serie de acciones que la mejorarán.
Las leyes constituyen el instrumento que va a permitir la coordinación entre los diferentes agentes, organizaciones, que intervienen en el deporte escolar. Las competencias, si las tienen, y responsabilidades vienen definidas en las normas legislativas de aplicación para cada una de ellas. Si hacemos un estudio competencial en el deporte escolar, comenzando por el Estado, observamos que la misma Constitución Española (1978) en el artículo 43.3, determina que los poderes públicos fomenten el deporte, por lo que parece normal que el Estado y el Consejo Superior de Deportes (C.S.D.), así como el resto de entidades públicas, tengan algún tipo de responsabilidad en esta materia, siempre dentro de su marco competencial.

La Ley del Deporte (10/90) fue promulgada, según Blanco (1999), para definir la competencia exclusiva del deporte en determinadas materias a nivel nacional. El C.S.D. es el organismo autónomo regulador del deporte a nivel estatal. Tiene atribuidas las funciones de la Administración General del Estado en materia deportiva. Entre sus competencias, marcadas en la Ley 10/90, artículo 8.j. está la de coordinar con las Comunida 
das en la Ley 10/90, artículo 8.j. está la de coordinar con las Comunidades Autónomas la programación del deporte escolar y universitario, cuando tenga proyección nacional e internacional.

Configurado el Estado de la Autonomías se ha limitado el papel del C.S.D. en la organización de la actividad física escolar. Su función es, según Burriel y Carranza (1995), la programación, dirección técnica y ejecución de los juegos y competiciones de carácter nacional o internacional correspondientes, quedando bajo la tutela de las Comunidades Autónomas el resto de tareas de organización y gestión del deporte escolar.

El complicado marco normativo actual que determina el sistema educativo en nuestro Estado viene configurado por la Ley reguladora del Derecho a la Educación (LODE) (1985), la Ley General del Sistema Educativo (LOGSE) (1990) y Ley Orgánica de Calidad de la Educación (LOCE) (2002). En el texto de las Leyes anteriormente citadas, han aparecido referencias a las actividades extraescolares, lo que va a definir la posibilidad de organización de las mismas por parte de la escuela.

Limitado el papel del C.S.D. en el deporte escolar como mero coordinador y responsable de las competiciones a nivel nacional y vista la posibilidad de organización de estas actividades por parte de los centros educativos, lógicamente en su espacio, parece claro que las Comunidades Autónomas son las que asumen la labor de fomento del deporte en edad escolar.

Las competencias de la Comunidad Aragonesa, comunidad objeto de nuestro estudio, vienen recogidas en la Ley del Deporte de Aragón (4/93) que tiene por objeto la ordenación, promoción y coordinación del deporte en el ámbito de la Comunidad Autónoma. En su artículo 3, que corresponde a las Disposiciones Generales, atribuye como competencia a las Administraciones Públicas de la Comunidad, la obligación de coordinarse para:

c) Colaborar en la enseñanza y programar la práctica de la actividad físico-deportiva escolar.

e) Promocionary atender especialmente las prácticas físico-deportivas de la juventud.

De lo cual puede deducirse que existe interés en la promoción, facilitación de estas actividades, sin hacer mención expresa sobre el fomento, es decir, acerca de la prestación del servicio deportivo escolar.

En Aragón, el Gobierno en materia deportiva utiliza la Dirección General de Deportes como órgano gestor del deporte en la Comunidad. La ley del Deporte de Aragón no atribuye a la Dirección General de Deportes en el artículo 7, ninguna competencia explícita sobre el deporte escolar.
Gobierno de Aragón, imputa mediante el Decreto 1904/2003, de 7 de julio, al Departamento de Educación, Cultura y Deporte la competencia sobre la promoción y fomento de la actividad deportiva en el ámbito de la Comunidad Autónoma, desarrollada a través de las entidades asociativas de carácter privado, clubes y otros entes deportivos, federaciones deportivas, y de entidades de carácter público, patronatos municipales y servicios comarcales de deportes, colaborando, así mismo, en la enseñanza y práctica de la actividad físico-deportiva escolar.

Bajo este marco jurídico, todos los años convoca los Juegos Escolares de Aragón, dirigidos a todos los centros de enseñanza públicos y privados, clubes deportivos, asociaciones y otras entidades sin ánimo de lucro, legalmente constituidas.

Continuado con las administraciones públicas, encontramos a los ayuntamientos, referencia más próxima del ciudadano en materia deportiva y el más directo (Blanco, 1995; Rebollo, 2000). El marco competencial de los ayuntamientos comienza con la Ley de Bases de Régimen Local (1985). En Aragón, como el resto de comunidades autónomas, continúa con la Ley del Deporte, que determina las competencias de las entidades locales. El artículo 8 enumera las competencias de los municipios en materia deportiva y el 9 regula los Centros de Coordinación Deportiva como instrumentos organizativos de ámbito inframunicipal, municipal y supramunicipal. De entre las competencias atribuidas a los municipios, en el ya mencionado artículo 8, ninguna de ellas hace mención expresa al deporte escolar o a las actividades físico deportivas dirigidas a los jóvenes. Si bien podemos pensar que tienen competencias en esta materia, puesto que se les atribuyen las siguientes responsabilidades:

b) Colaborar con la ejecución de programas para la promoción de las actividades físico deportivas, de acuerdo con los criterios que determine la Diputación General de Aragón.

e) Organizar o colaborar con la organización de competiciones deportivas de ámbito municipal, sin perjuicio de las competencias atribuidas a las Federaciones Deportivas Aragonesas.

Esa suposición realizada anteriormente se basa en el concepto de promoción. Este concepto nos acerca a unos programas que responden, en general, a criterios de interés, adecuación y formación de las personas, en los cuales creemos que deben incluirse necesariamente los niños en edad escolar.

Los ayuntamientos, donde sí de forma explícita, tal y como marca la LOGSE en el artículo 57.5.y también el artículo 69.2 de la LOCE, tienen algún tipo de responsabilidad en el deporte escolar desarrollado en el marco de la escuela: las Administraciones locales podrán colaborar con los centros 
educativos para impulsar las actividades extraescolares y promover la relación entre la programación de los centros y el entorno socioeconómico en que éstos desarrollan su labor. Desgraciadamente, una gran parte de los centros educativos han dado la espalda a cualquier tipo de intromisión en este espacio pedagógico, lo que ha originado que una gran parte del deporte escolar se desvincule de la escuela.

El papel de los Centros de Coordinación deportiva o Servicios Comarcales de Deportes (S.C.D.) es muy importante en la gestión del deporte aragonés. Lo es hasta tal punto que, a juicio de Bermejo (1995), a pesar de que los municipios cuenten con el reconocimiento constitucional y legal para el desarrollo de atribuciones en materia deportiva, carecen en muchas ocasiones de la posibilidad real de llevar a cabo sus competencias. Razón por la cual, la Ley del Deporte de Aragón apuesta por los S.C.D. como los foros más apropiados para el desarrollo de las competencias deportivas en el ámbito municipal. Las competencias de los S.C.D. vienen definidas en el artículo 9.3. de la Ley del Deporte de Aragón. Sólo en una de las competencias se hace referencia a las actividades escolares y la variante de éstas que se menciona es la competitiva: Organizar las competiciones deportivas escolares dentro de su ámbito territorial.

Se entiende, tras una lectura global y completa del mencionado artículo, que los S.C.D. tienen como función la de asesorar y facilitar las actividades físico deportivas organizadas por los ayuntamientos, haciendo llegar éstas al mayor número posible de ciudadanos, lo que incluye necesariamente a los niños y niñas en edad escolar.

El marco legislativo de los S.C.D. no finaliza aquí, encontramos que la Ley de Comarcalización (23/2001) determina en su art. 10, que éstos tienen entre sus competencias la ejecución de programas de fomento de la enseñanza y práctica de la actividad físico-deportiva escolar y juvenil, con especial incidencia en la difusión de los deportes tradicionales aragoneses. Junto a ello, poseen la potestad de la organización y colaboración en la organización de competiciones deportivas de ámbito comarcal, en especial las fases comarcales de los Juegos Escolares de Aragón. Todo ello sin perjuicio de las competencias atribuidas a las federaciones deportivas aragonesas y a las entidades locales.

Vistas las competencias de las distintas administraciones o entidades públicas en materia de deporte escolar en la Comunidad Aragonesa, pasemos ahora a ver las competencias que la Ley del Deporte de Aragón determina para cada una de las entidades deportivas no públicas.

Las asociaciones deportivas aragonesas que tienen como fin la práctica deportiva de sus asocia- dos, pueden ser calificados según la Ley del Deporte de Aragón como Clubes Deportivos o Federación Deportiva (artículo 15, de la Ley 4/93). Los clubes deportivos aragoneses son asociaciones privadas, integradas por personas físicas o jurídicas, que tienen por objeto principal la promoción y el desarrollo de actividades físico deportivas, o la práctica de una o más modalidades deportivas por sus miembros, o la participación en actividades o competiciones deportivas de carácter oficial, profesional o aficionado (artículo 17, de la Ley 4/ 93).

La Ley del Deporte de Aragón por lo que se refiere a las competencias de los clubes en el deporte escolar no hace referencia alguna, dejando claro que las atribuciones de éstos son la promoción y el desarrollo de actividades físico deportivas o modalidades deportivas por parte de sus asociados, sin distinguir en función de la edad o el nivel.

Las Federaciones Deportivas Aragonesas, definidas en el artículo 24 de la Ley del Deporte de Aragón como entidades privadas, con personalidad jurídica y patrimonio propios, integradas por clubes deportivos, técnicos, jueces y árbitros, deportistas, y en su caso, agrupaciones de clubes deportivos, que, además de sus propias atribuciones, ejercen por delegación de la Comunidad Autónoma, las funciones de promoción y desarrollo ordinarios del deporte en el ámbito territorial aragonés.

Como función principal, para nuestro objeto de estudio, el artículo 26 de 4/93, atribuye a las Federaciones la organización o tutela del desarrollo de actividades y competiciones deportivas. Al igual que ocurre con los clubes, las Federaciones no tienen ninguna referencia explícita al deporte escolar. Son las encargadas, en corresponsabilidad con la Administración Pública Local en Aragón que tiene las competencias en materia deportiva escolar, de estructurar y organizar las actividades de su modalidad deportiva ya sean de promoción o de competición, acomodándolas a los objetivos que corresponden a la edad escolar.

\section{Metodología}

El objeto del presente artículo es conocer la imagen que las diferentes organizaciones, públicas y privadas, con competencias en deporte escolar, tienen para los colectivos implicados: alumnos, padres y técnicos deportivos, en la Comarca Ribera Baja del Ebro. Dentro de este objetivo general podemos concretar en el conocimiento de:

- Imagen de las organizaciones educativas:

* Centros escolares

* Docentes de las escuelas 
- Imagen de las administraciones públicas:

* Ayuntamientos

* Servicio Comarcal de Deportes

* Gobierno de Aragón

- Imagen de las Federaciones deportivas aragonesas.

La investigación se realizó en cuatro grupos de población ubicados en el ámbito geográfico mencionado anteriormente más el municipio Fuentes de Ebro. Los grupos de población objeto de estudio son:

1. Los/as niños/as de último ciclo de Primaria y Secundaria Obligatoria que viven en los $10 \mathrm{mu}-$ nicipios que integran el S.C.D. y el municipio Fuentes de Ebro.

2. Los/as técnicos deportivos de las distintas modalidades que trabajan con los niños/as entre 10 y 16 años.

3. Los padres y madres de los/as niños/as que asisten a las escuelas deportivas.

4. Los responsables de la gestión de los programas de actividades físicas dirigidas a los niños y niñas de 10 a 16 años.

Con el fin de mejorar la investigación social se introdujo el concepto de combinación, entendido como la asociación de métodos o técnicas en el estudio del mismo fenómeno. En nuestra investigación utilizaremos el multimétodo de combinación, puesto que esta estrategia se basa en la idea de que el resultado obtenido en una investigación, que ha aplicado un método, puede perfeccionar la implementación de algún componente o fase de la misma realizada con otro método, logrando incrementar la calidad de los resultados obtenidos (Bericat 1998).

La primera técnica utilizada es el cuestionario. Debido al número de grupos objeto de estudio, confeccionamos tres diferentes, optando por modalidades diferentes de aplicación:

- Cuestionario de niños y niñas entre los 10 a 16 años, auto-administrado de aplicación masiva, por aulas y con la presencia del encuestador. El número de cuestionarios recogidos después del trabajo de campo fue de 624,0 lo que es lo mismo el $100 \%$ de la población.

- Cuestionario de padres y madres de niños y niñas, optamos por dos tipos: cuestionario autoadministrado sin presencia de encuestador y por cuestionario estandarizado con presencia del encuestador. En este segundo grupo de población recogimos 146 encuestas, lo que supone un $45 \%$ de respuesta. Por lo que podemos afirmar que trabajamos con un error de $\pm 6,14$ y un nivel de confianza del 95,5\%.
Cuestionario de monitores y monitoras de las escuelas deportivas, hemos optado por un cuestionario auto-administrado con la presencia del encuestador. Este grupo está formado por tan sólo 16 personas, obteniendo un índice de repuesta del $100 \%$.

Realizado el trabajo de campo se analizaron los datos, se tabularon y mecanizaron, mediante el paquete informático SPSS 12.0, que posibilitó la puesta en práctica de las técnicas estadísticas precisas para esta investigación.

En primer lugar, se recurrió a un análisis de tipo descriptivo. Tras éste pasamos al análisis inferencial que tuvo por objeto constatar el grado de asociación/relación entre las diversas variables controladas.

La segunda técnica utilizada fue el grupo de discusión. Para Benedito (1977) es un intercambio de ideas y opiniones de un grupo reducido de personas que tienen interés común para discutir sobre un tema. Según Krueger (1991: 46) los miembros de un grupo de discusión interaccionan y comparten una información que posiblemente no podríamos obtener por medio de entrevistas individuales, cuestionario u otras técnicas de recogida de datos.

El grupo de discusión es una técnica de investigación social que, según Ibáñez (1992), posee mayor potencia metodológica y epistemológicamente es posterior que la encuesta, lo que le convierte en una técnica más progresiva. De igual manera, la discusión en grupo tiene una función consuntiva, donde los individuos expresan su vinculación con el sistema. Esta técnica hace una combinación de elementos de otras técnicas como serían la entrevista y la observación participante (Morgan, 1988: 15).

En la composición del grupo de discusión optamos por una composición simétrica no coherente, y además heterogénea, a fin de que el grupo con estas características nos aporte mayor riqueza en la producción del discurso y, por lo tanto, en los datos aportados, resultando un grupo de siete personas.

\section{Resultados}

Imagen de las organizaciones educativas. Centros escolares y docentes.

Para el $87,5 \%$ de los técnicos deportivos, no existe ninguna relación entre las escuelas deportivas, deporte extraescolar, y los centros educativos. Sólo el $12,5 \%$ de los técnicos opina que sí que existe esta relación.

La mayor parte de los padres $(76,9 \%)$ creen que desde los centros educativos se motiva a los alum- 
nos a realizar actividad física extraescolar. Son más los padres y madres que creen que los docentes de Educación Física invitan a realizar estas prácticas $(79,7 \%)$.

En opinión de los técnicos $(62,5 \%)$ los profesores de los centros educativos motivan a los niños a realizar actividad física en su tiempo libre y, casi un tercio de los técnicos se manifiesta en contra de esta posibilidad. Para los técnicos $(68,8 \%)$, los docentes de Educación Física conocen cuantos alumnos participan en las escuelas y equipos deportivos.

Existe una relación estadísticamente significativa entre el centro educativo y la respuesta de sus alumnos a la motivación de los docentes ( $P=.000)$. interesa el dominio de una serie de conocimientos y capacidades, por parte de los alumnos, que le van a permitir pasar de ciclo o etapa. La escuela es un centro donde se transmiten conocimientos y no participa, en este momento, de la educación global de la persona, pues ante cualquier dificultad, en este caso de tipo organizativo, se desmarca.

Debería producirse un cambio institucional, creo que los ayuntamientos estarían dispuestos, pero Educación no lo sé (Coordinador 1)

Por parte del I.E.S. de Fuentes se ha intentado hacer una actividad extraescolar por las tardes, con el inconveniente de los desplazamientos (Coordinador 2).

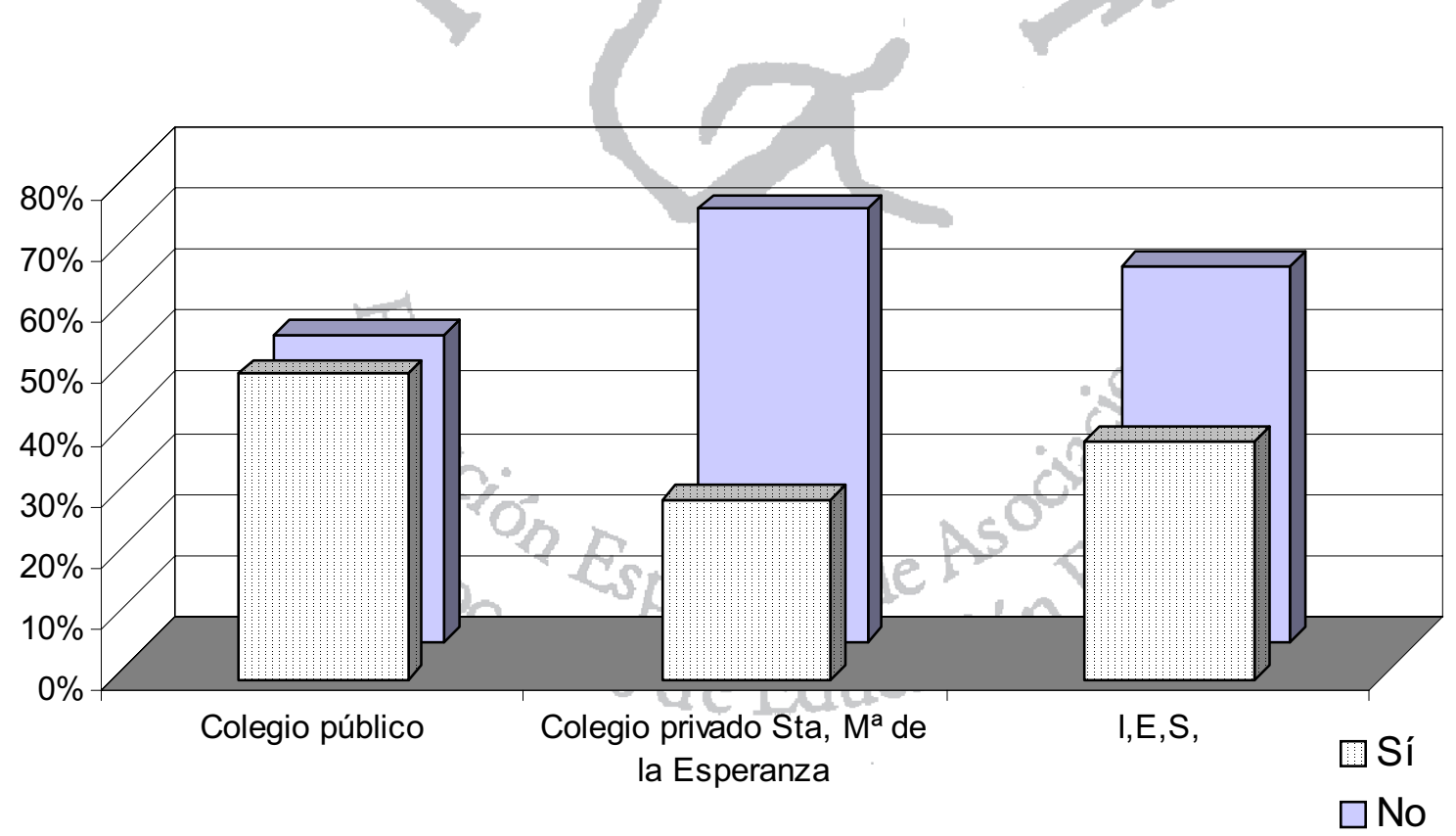

Figura 1. Motivación a realizar deporte en función de los centros educativos

En los centros públicos de Educación Primaria de cada localidad, es donde más alumnos afirman que los docentes les motivan para que hagan deporte en su tiempo libre (49,8\%). El colegio privado es el centro donde menos alumnos piensan que los docentes les motivan (29,3\%) (figura 1).

El $70,9 \%$ de los niños y niñas inscritos en actividades físicas organizadas afirman que sus profesores de Educación Física saben que están realizando este tipo de actividades. Existe relación $(P=.000)$ entre el ciclo educativo y esta opinión, puesto que son más alumnos de Primaria $(90,2 \%)$ los que así lo manifiestan, que el alumnado de Secundaria $\left(62,6 \% 1^{\circ}\right.$ ciclo de la ESO y $57,7 \% 2^{\circ}$ ciclo de la ESO).

Según el grupo de discusión, la institución escolar está desligada completamente de la formación extraacadémica de los alumnos/as. La escuela no se interesa por este tipo de educación, le

\section{Imagen de las administraciones públicas.}

Los padres $(74,8 \%)$ de los alumnos que asisten a escuelas deportivas, opinan que las administraciones, en general, no colaboran suficientemente con el deporte escolar. Por su parte, los técnicos piensan que la administración que más ayuda al deporte escolar es la municipal, puesto que el $62,5 \%$ de los técnicos cree que el apoyo es bastante. Un $57,14 \%$, sostiene que el apoyo del S.C.D. es bastante. Algo más del setenta por ciento, no está de acuerdo con la labor del Gobierno de Aragón, el $43,8 \%$ cree que es poco y un $31,3 \%$ piensa que es nada lo que colabora con el deporte escolar. Las Federaciones deportivas son también puestas en tela de juicio, pues el $60 \%$ de los técnicos cree que hacen poco y un $6,6 \%$ afirman que es nada (figura 2).

Analizando una por una las administraciones con competencias en el deporte escolar encontramos en el lugar más próximo a los ciudadanos a los ayun- 


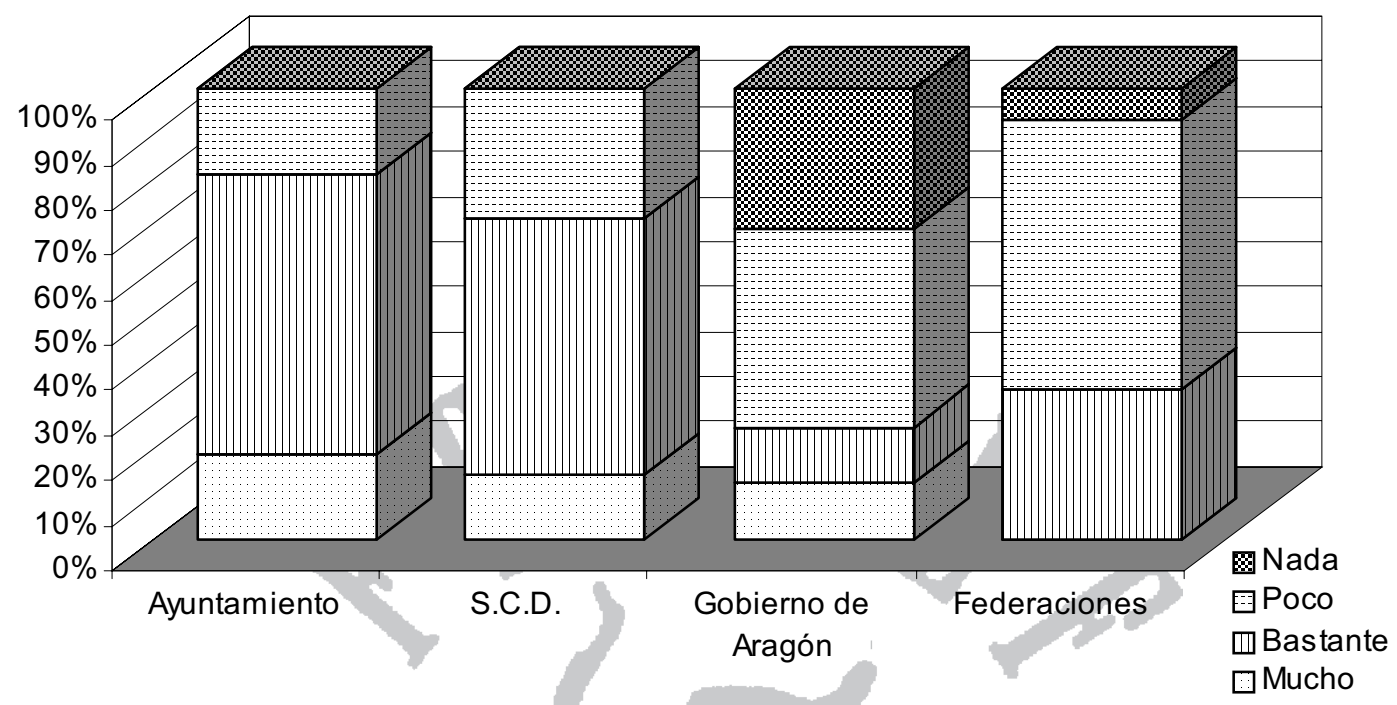

Figura 2. Valoración del apoyo de las diversas entidades en el deporte escolar

tamientos. Son las instituciones que repercuten de una forma más directa en los usuarios de las actividades. Los técnicos (81\%) están bastante satisfechos con la gestión de sus municipios, pues según ellos, apoyan mucho o bastante al deporte de base. Un 19\% de los técnicos piensa que su ayuntamiento participa poco en el deporte escolar. El deporte escolar es en nuestro ámbito un producto de las administraciones públicas locales.

Los políticos, en nuestros ayuntamientos, responden a dos tipos de estímulos: los personales y los electorales. Ambos condicionan la política deportiva de los respectivos municipios, que en muchos casos no está definida y se limita a producir actividades o acontecimientos sin objetivo preciso

Debería producirse un cambio institucional, creo que los ayuntamientos estarían dispuestos, pero Educación no lo sé. Más que nada por que los concejales tienen hijos y les interesa (Coordinador 1).

Nos han puesto alli el pabellón de las fiestas $y$ nos han fastidiado, por que yo entiendo que se tenga que usar el pabellón para bailar, pero que te fastidien durante quince días, no me parece bien (Técnico 2).

Esta falta de claridad política se traduce en la nula participación de los técnicos y lo que es peor, de los alumnos, en diversas actividades. Situación agravada por el afán o interés competitivo que preside la política deportiva municipal.

¿Por qué los críos cuando hacemos atletismo no van, en el caso de muchos pueblos? (Coordinador 1).

¿Habría pueblos que vendrían a participar a balonmano y baloncesto? (Coordinador 1).
Entonces esos pueblos no ganarían, que es lo que quieren (Lcdo. E.F.1).

Esta filosofía o situación se manifiesta especialmente en un municipio, $D$, que como se pudo observar en el análisis del cuestionario se caracteriza por la escasa participación, por contar con los técnicos de menor nivel académico y deportivo y por disponer de la peor coordinación de todas las escuelas. Esto no pasaría de ser una mera anécdota, sino fuese por que es la población del Servicio Comarcal de Deportes con mayor número de personas y porque la contratación del técnico responsable del Servicio se hace a cargo del Ayuntamiento de D.

Eso está en la filosofía del pueblo, en la gente. La filosofía es ganar. En ese pueblo desde el padre hasta el crío (Lcdo. E.F. 1).

Pienso que hay cosas que son muy difíciles de cambiar, son cosas que están muy metidas en el pueblo (Lcdo. E.F. 1).

Es que es una cosa de chicos, padres, monitores, ayuntamiento... Es una cosa muy extendida (Lcdo. E.F. 1).

El Servicio Comarcal de Deportes es la siguiente organización pública que tiene competencias en la organización del deporte escolar en nuestro ámbito de estudio. El apoyo que esta organización presta al deporte escolar es calificado de mucho por el $14,28 \%$ de los técnicos, de bastante por el $57,14 \%$ y de poco por el $28,57 \%$.

El técnico responsable está atado de manos (la contratación depende directamente del ayuntamiento D), no pudiendo desarrollar con normalidad su trabajo. La frustración llega hasta tal punto que pide ayuda al resto de técnicos deportivos. 
Yo como coordinador me veo atado, no puedo decir que todos fuera, necesito el apoyo de un grupo (Coordinador 1).

Yo creo que tú, Andrés, a nivel de coordinador deberías redactar los objetivos y de esa forma determinar la filosofía (Lcdo. E.F. 1).

La última administración pública con competencias en materia deportiva es el Gobierno de Aragón y más concretamente la Dirección General de Deportes. Para ningún técnico el apoyo del Gobierno de Aragón es mucho, considerando, tan sólo el $33,3 \%$ de los técnicos que es bastante, el $60 \%$ piensa que es poco y el 6,6\% restante afirma nada.

Por proximidad al ciudadano y por política descentralizadora, la institución pública más alejada es el Gobierno de Aragón. El papel de las administraciones cada vez va a ser menor en el deporte, hecho que se cumple en Aragón. La Administración autonómica ha delegado en las Federaciones deportivas.

La implicación del Gobierno de Aragón es muy limitada y con una filosofía competitiva (Coordinador 1).

Por lo que concierne a las actividades físico deportivas extraescolares para niños, el Gobierno de Aragón solamente subvenciona la competición, los Juegos Escolares. Todo 10 que se sale de Juegos Escolares, ya forma parte de actividades generales que no son subvencionadas (Coordinador 1).

Imagen de las federaciones deportivas aragonesas.

Las federaciones deportivas son una de las entidades que peor paradas resultan de la opinión de los técnicos. El $75 \%$ de ellos opinan que apoyan poco o nada al deporte de base. El $25 \%$ restante se distribuye entre mucho y bastante.

\section{Discusión y conclusiones}

Creo que es necesario resaltar en primer lugar, aunque parezca extraño, que las normas aragonesas no hacen referencia directa, exceptuando algunas ocasiones, al deporte escolar o a las escuelas deportivas. La intención de los legisladores, políticos y responsables de la gestión deportiva en el territorio aragonés ha sido la de descentralizar el desarrollo y organización de estas actividades (DGA, 1985). Se ha pretendido que la administración local, ayuntamientos y comarcas, asumiera la materialización de estos programas por su proximidad con el ciudadano, para favorecer el desarrollo del sistema deportivo aragonés. Verdaderamente lo han conseguido.
De las instituciones públicas, los ayuntamientos son la referencia más próxima del ciudadano en materia deportiva, (Blanco, 1995; Rebollo, 2000), son los prestadores reales del servicio. Los técnicos son más benévolos con la labor de estas instituciones que con el resto. Cuanto más próxima es la institución al técnico, mejor valorada resulta. Así el ayuntamiento es la organización pública menos criticada, seguido por el S.C.D. Mientras que Gobierno de Aragón y Federaciones, organizaciones que actúan por delegación del Gobierno, son las organizaciones más criticadas entre éstos.

Aclararemos que debido a la escasa implicación de los progenitores en el deporte escolar de sus hijos, les preguntamos de forma general, no organización por organización, tal y como hicimos con los técnicos. Son pocos los padres de los alumnos que asisten a escuelas deportivas, los que opinan que las administraciones, en general, colaboran suficientemente. Por esta razón y por los resultados obtenidos entre los técnicos, sería interesante, para su imagen, que las diferentes instituciones realizasen, además de un buen trabajo de gestión del deporte escolar, mayor labor divulgativa de las acciones dirigidas a este grupo de edad. El Gobierno de Aragón debe replantearse su política en este campo, pues a pesar de ser la organización que financia estas actividades, en la vertiente competitiva, su actuación no es valorada positivamente

En relación a los centros escolares, a pesar de la «buena» opinión que padres, técnicos y escolares, tienen de los docentes, la realidad es que la institución para la que trabajan no se implica, como muy bien han dicho la mayor parte de los técnicos, en este tipo de actividad extraescolar. Éste es uno de los grandes puntos débiles del deporte escolar.

Continuando con los puntos débiles encontramos a las organizaciones privadas. En relación con las de primer grado, clubes, sólo diremos que apenas existen en este ámbito geográfico y no tienen ninguna actuación en el deporte escolar.

En último lugar hemos dejado a las Federaciones que actúan por delegación del Gobierno de Aragón. Son las organizaciones peor valoradas por los técnicos. Éstas, al igual que la administración autonómica, deben realizar un esfuerzo para la mejora de su imagen.

\section{Bibliografía}

Bermejo, J. (1995). La Ley del Deporte de Aragón y los municipios: desarrollo normativo. En Diputación General de Aragón (Edita). Cuadernos técnicos del deporte $n^{\circ} 21$. XII Seminario Aragonés Municipio y Deporte. La Ley del Deporte y los municipios aragoneses. Zaragoza: Diputación General de Aragón. pp. 11-14. 
Bizquerra, R. (1987). Introducción a la estadística aplicada a la investigación educativa. Barcelona: PPV.

Blanco, E. (1995). Análisis comparativo de las competencias del deporte en las diferentes administraciones públicas. En Diputación General de Aragón (Edita). Cuadernos técnicos del deporte $n^{\circ}$ 23. XIII Seminario Aragonés Municipio y Deporte. Zaragoza: Diputación General de Aragón. pp. 9-16.

Blanco, E. (1999). El marco conceptual. En Blanco, E., Burriel, J.C., Camps, A., Carretero, J.L., Landaberea, J.A., y Montes, V.(Eds.). Manual de la organización institucional del deporte. Barcelona: Paidotribo. pp. 19-30.

Burriel, J. C., y Carranza, M. (1995). Marco organizativo del deporte en la escuela. En Bláquez, D. (Ed.). La iniciación deportiva y el deporte escolar. Barcelona: Inde. pp. 431-448.

Diputación General de Aragón. (1985). Actividad deportiva infantil. Curso 1985/86. Zaragoza: Diputación General de Aragón.
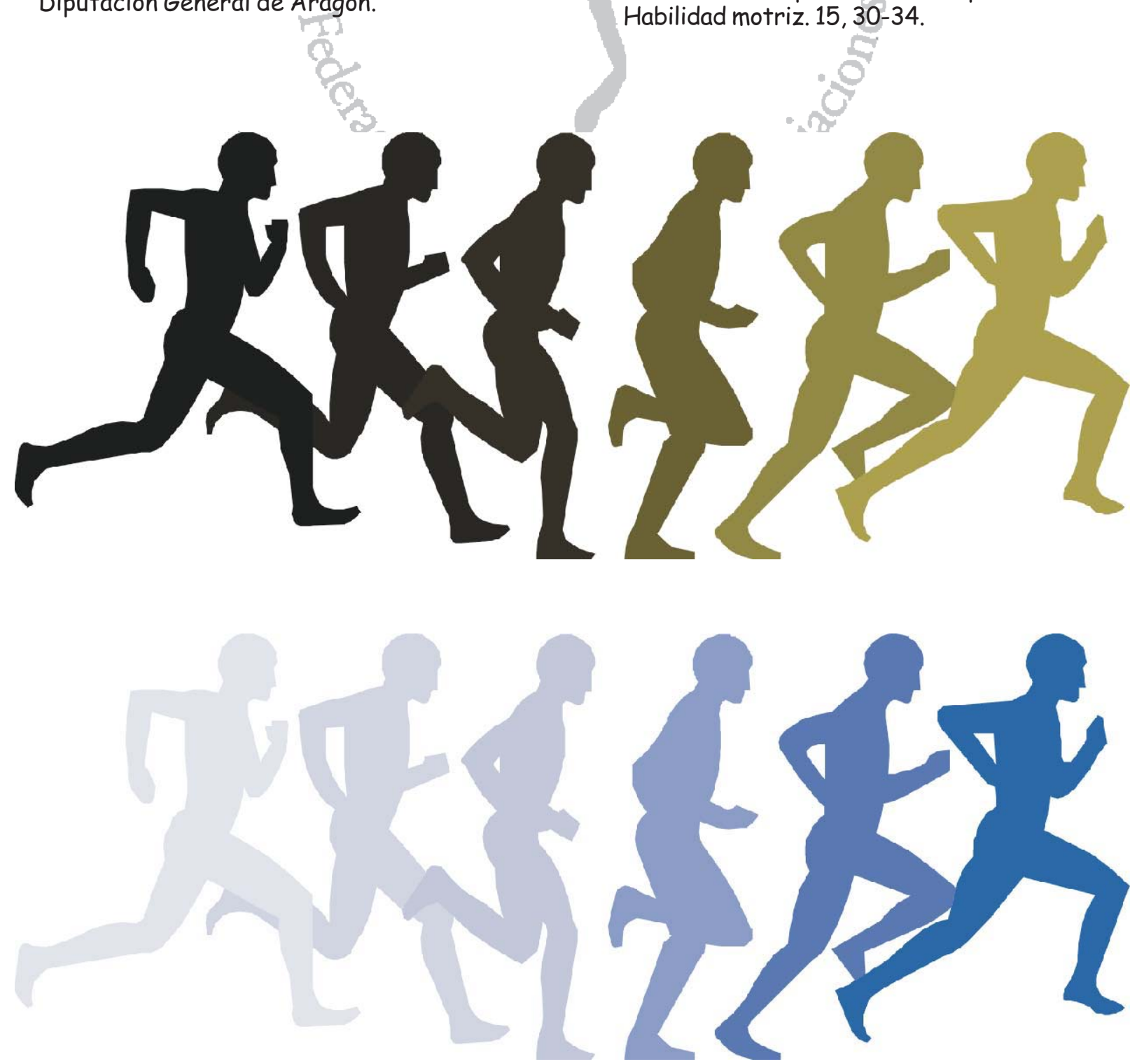